\title{
Termas de la Garita
}

\author{
Alejandro Berrocal Murillo
}

Reseña de proyecto académico

Taller de Diseño X Arquitectura Tropical. Año 2017 Universidad de Costa Rica - UCR

Laboratorio de Arquitectura Tropical - LAT

Universidad de Costa Rica - UCR

Profesores: Arq. Eugenia Solís y Arq. Emily Vargas

alejandrojosueberrocal@hotmail.com

Recibido: 2 de julio del 2017

Aceptado: 4 de agosto del 2017

\section{Resumen:}

Termas de la Garita es un proyecto académico del Taller de Diseño opción Arquitectura Tropical, del último nivel de la Carrera de Arquitectura de la Universidad de Costa Rica (UCR). Este se desarrolló en conjunto con el Laboratorio de Arquitectura Tropical (LAT) de la Escuela de Arquitectura para un control importante de las variables bioclimáticas del proyecto que contempla un complejo de termas en una zona de clima cálido-húmedo de Costa Rica.

Palabras clave: arquitectura; proyecto académico; termas.

\begin{abstract}
:
Termas de la Garita is an academic project of the Design Workshop (Tropical Architecture Option) in the last year of the Architecture Major in the University of Costa Rica (UCR). This project was developed along with the Laboratory of Tropical Architecture (LAT) of the School, in order to have an effective control of the bioclimatic variables of the project, because it takes place in a warm-humid zone of Costa Rica.
\end{abstract}

Keywords: architecture; academic project; spas

\section{Alejandro Berrocal Murillo}

Estudiante de Taller de Diseño X Arquitectura

Tropical. Año 2017. Universidad de Costa Rica UCR. 
I proyecto Termas de La Garita está ubicado en La Garita de Alajuela,Costa Rica. El complejo de termas enfatiza, dentro de su programa arquitectónico, las condiciones interiores de confort para el usuario en un clima cálido-húmedo, el manejo plástico e iluminación del espacio interior, siempre en contemplación del entorno natural en el cual se proyecta, buscando la armonía y el respeto con el paisaje.

Partiendo de los conceptos que tienen que ver con lo estereotómico, y haciendo de este el principal camino para lograr una transmisión y continuidad entre el objeto arquitectónico y el terreno natural que lo contiene; tomando las raíces del entorno y desarrollando su crecimiento a partir de los rayos de luz que perforan a la masa, y que ponen en evidencia el sentido del espacio.

El proyecto se plantea en base a dos terrazas o niveles, en donde la luz es la encargada de configurar el objeto; una primer terraza que se introduce en el terreno, realzando el sentido de cueva y la segunda terraza que apenas sobresale de la superficie escalonada del contexto, formas puras, entrantes y salientes que se insertan en la topografía. Con estas premisas se da partida a la búsqueda del desarrollo de una propuesta concreta que minimice el impacto visual, proyectándose ligeramente sobre la superficie, con un elemento longitudinal a modo de cubierta y que da la continuidad al objeto.

El programa lo componen cinco grandes áreas, que completan 1900 metros cuadrados propuestos; la primer gran área referente a las aguas, con dos piscinas y cinco estanques a temperaturas específicas para el disfrute de los bañistas; una segunda área de esparcimiento que las dividen cuatro terrazas externas y un espacio para la alimentación del complejo termal. La tercer área corresponde al espacio de terapias, cámaras de masajes individual y, en pareja, baños de vapor y tratamientos de belleza, son parte de las actividades que se albergan en este bloque y que se conectan con la cuarta área, un espacio para el desarrollo espiritual y sentido de relajación, yoga grupal e individual, que se conectan con el exterior y la naturaleza del terreno. Por último, el área administrativa y de aprovisionamiento, que se disponen en el proyecto como pequeños núcleos cerrados.

A lo interno del proyecto logra apreciarse cómo las perforaciones logran convertirse en los pequeños pretextos que dan sentido al funcionamiento de lo complejo, aperturas a la luz que alimentan la riqueza de los espacios y que sensibiliza la rudeza del material.

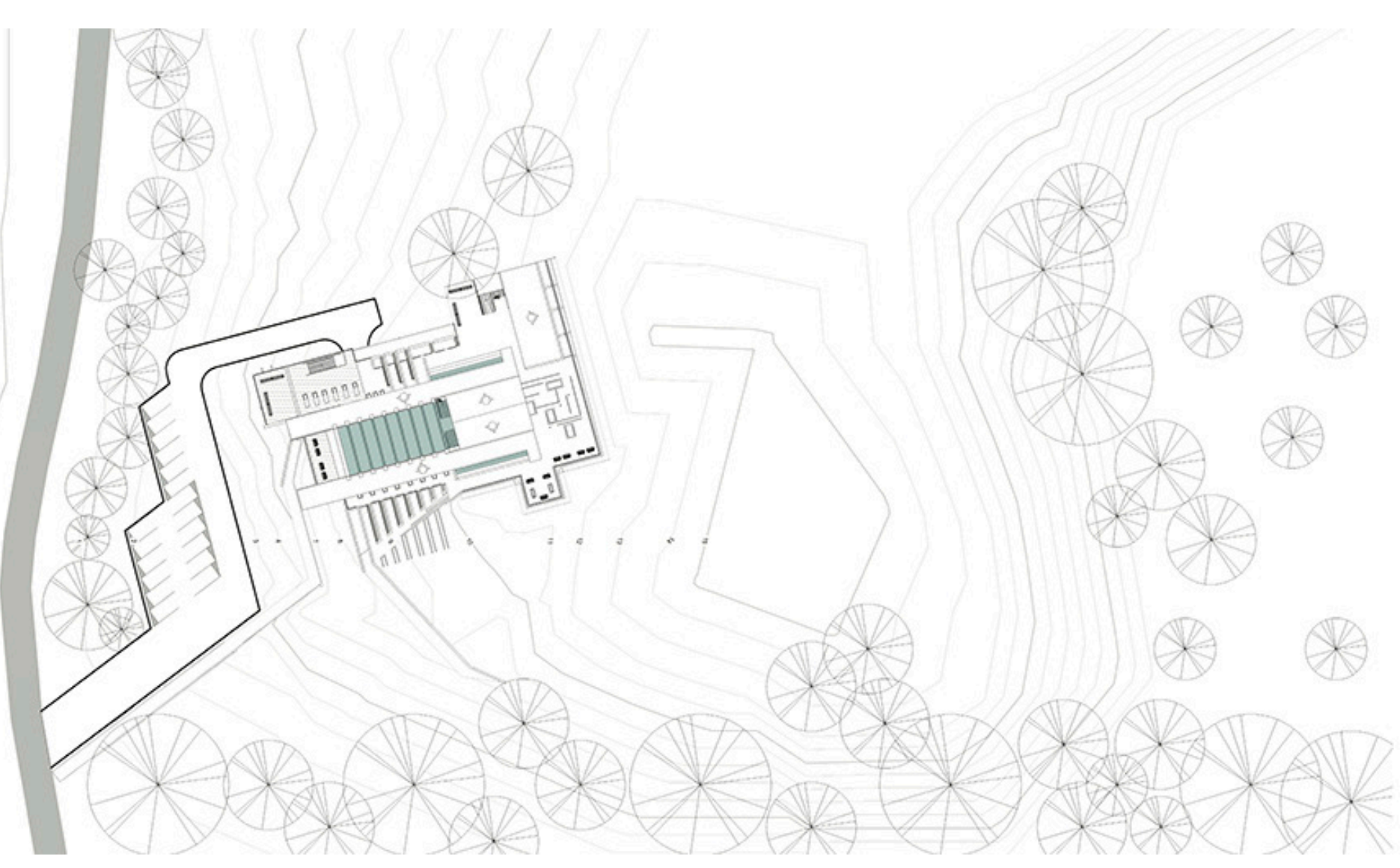




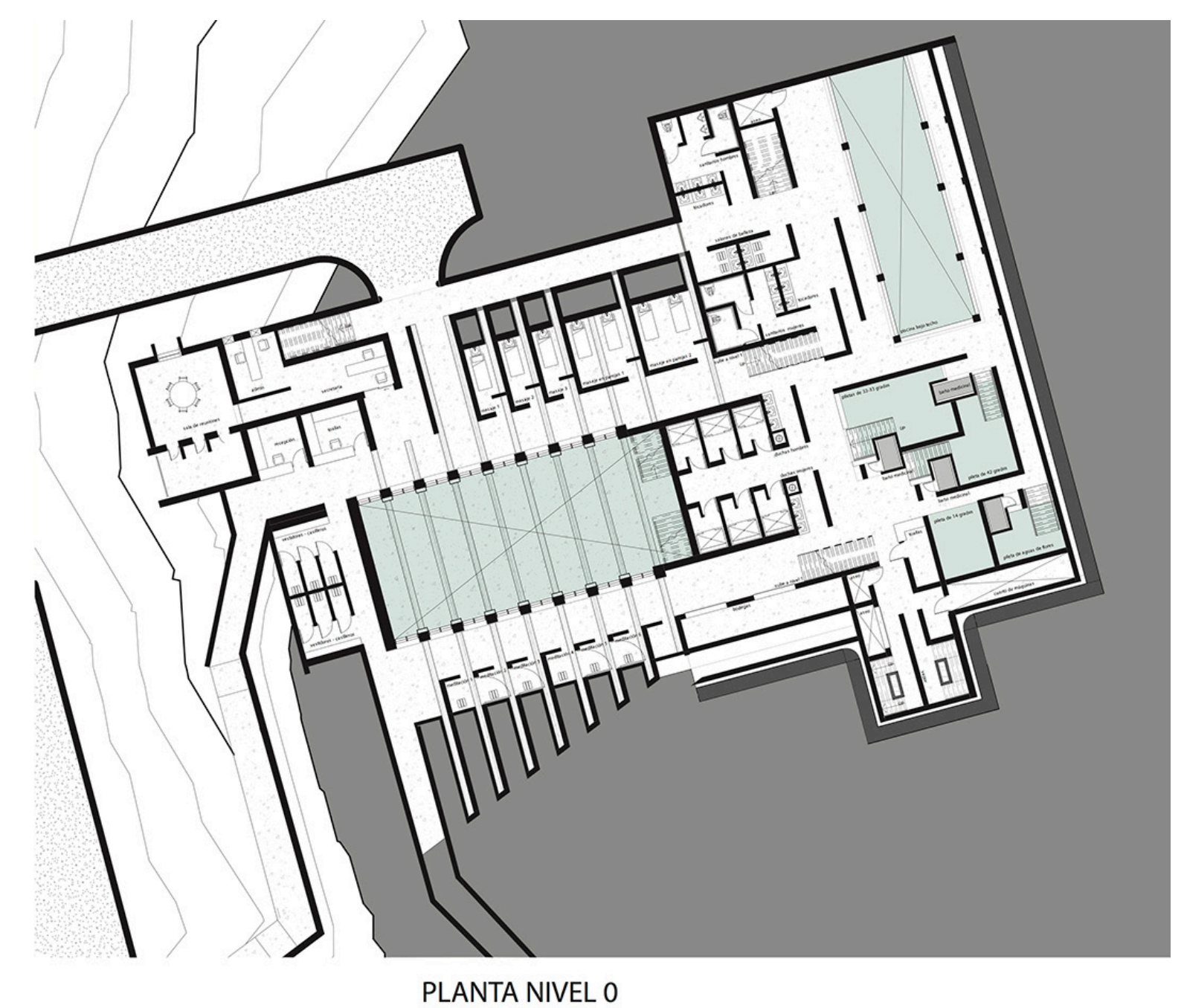

Figura 2. Plantas arquitectónicas Termas de la > Garita

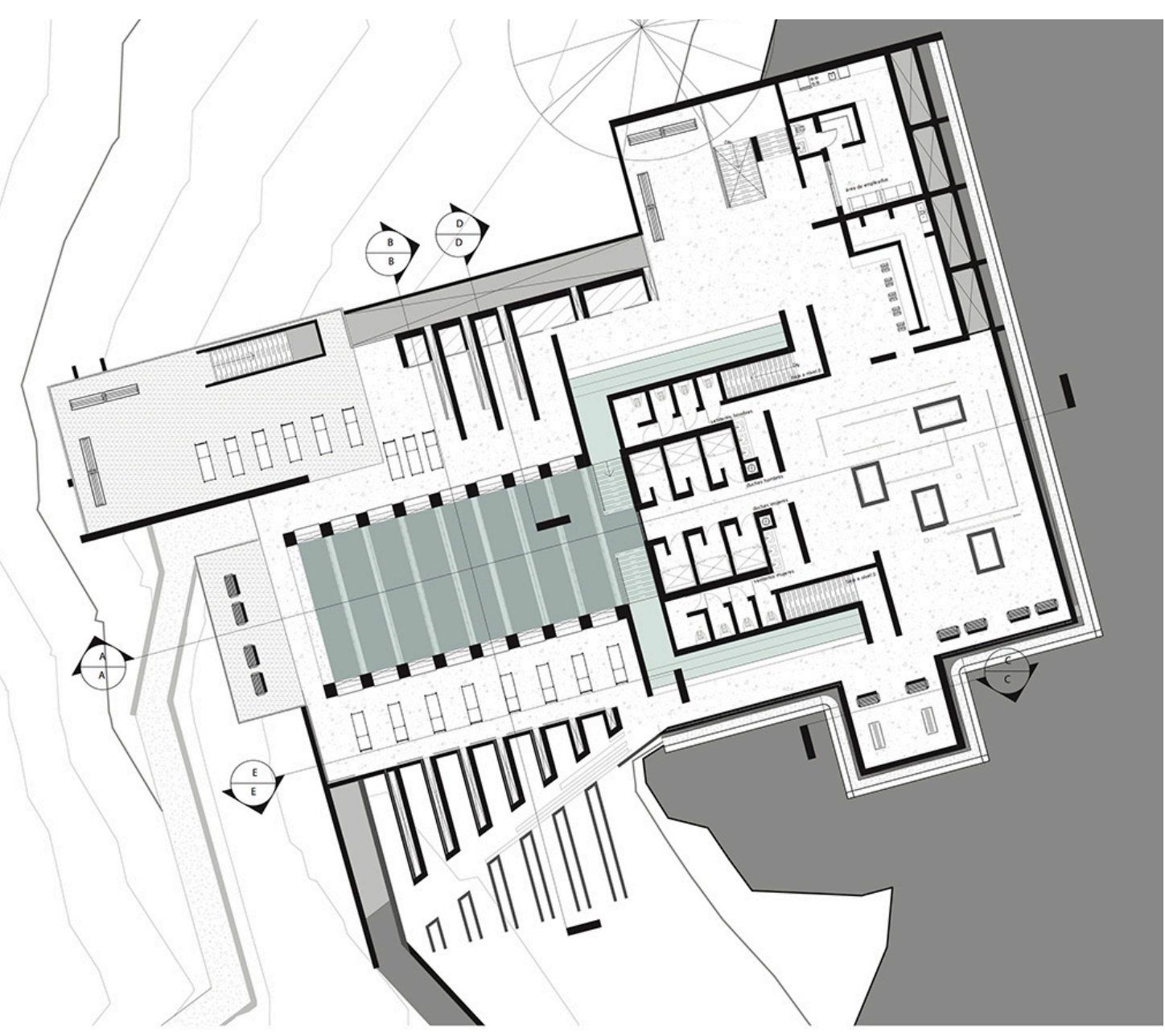

PLANTA NIVEL 1

Figura 3. Elevación arquitectónica Termas de la $Y$ Garita

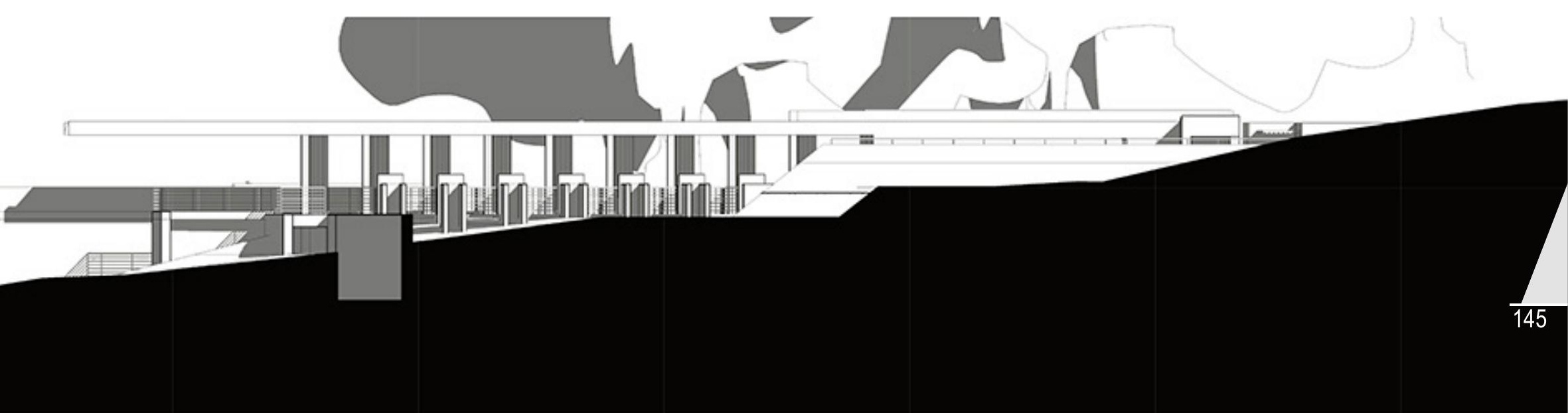



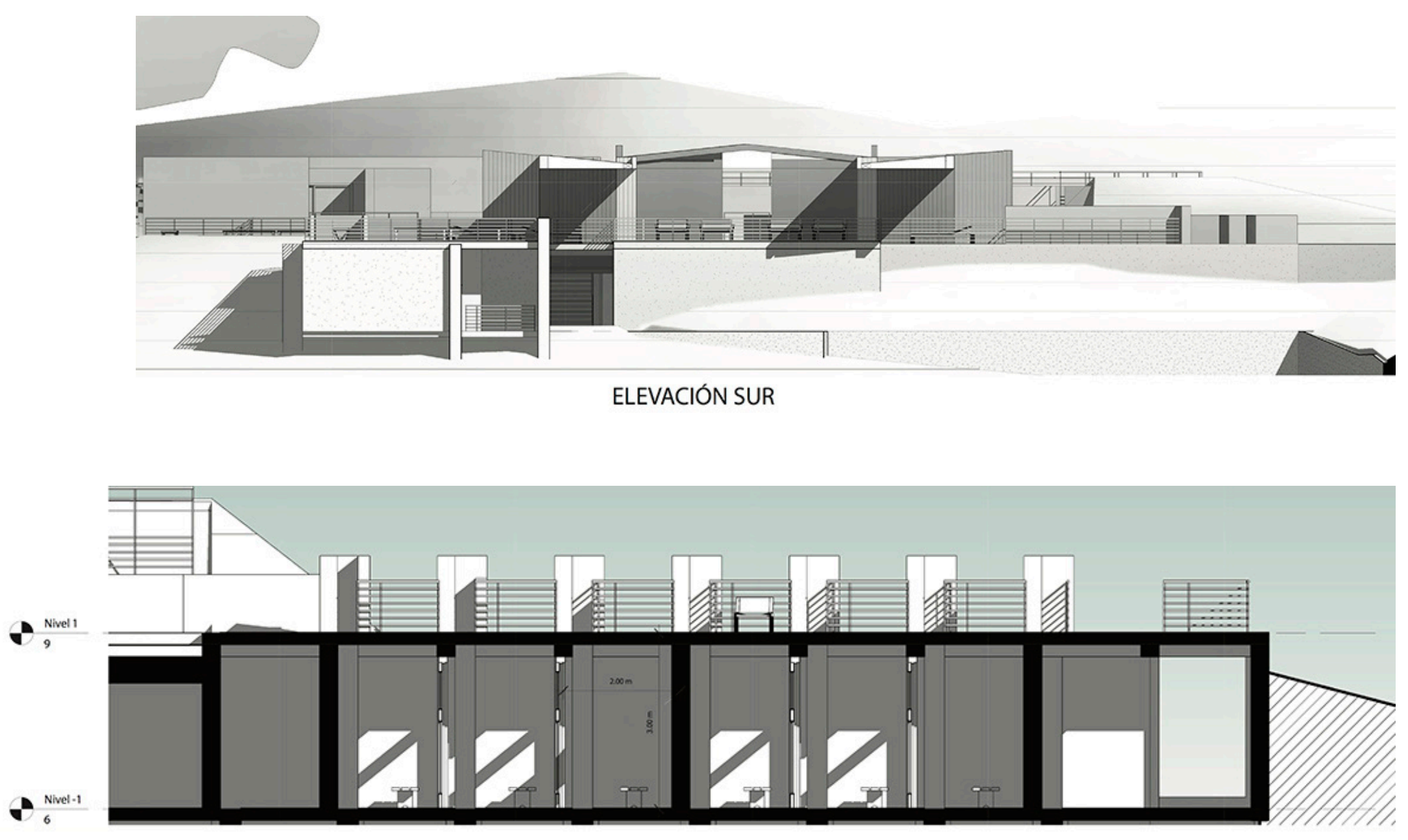

SECCIÓN E-E

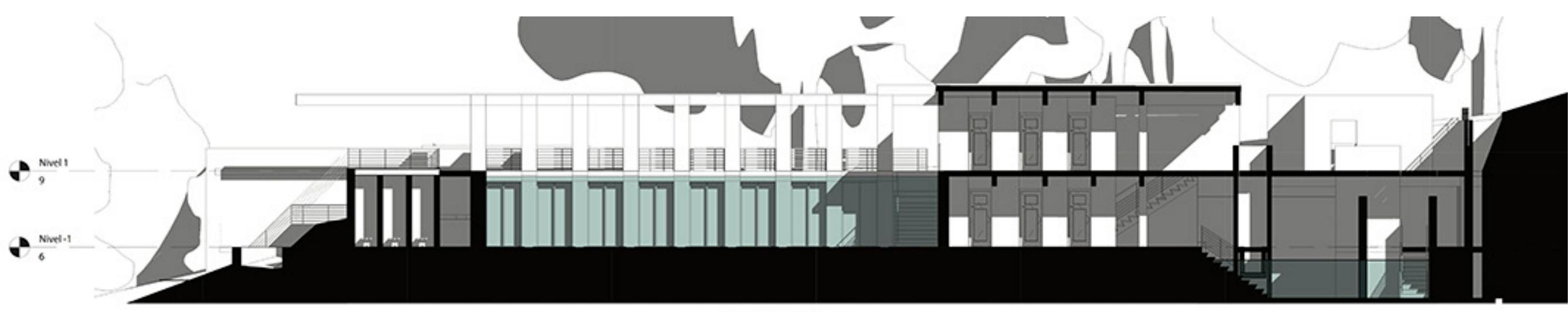

SECCION A-A
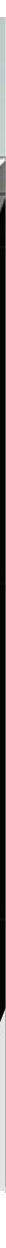


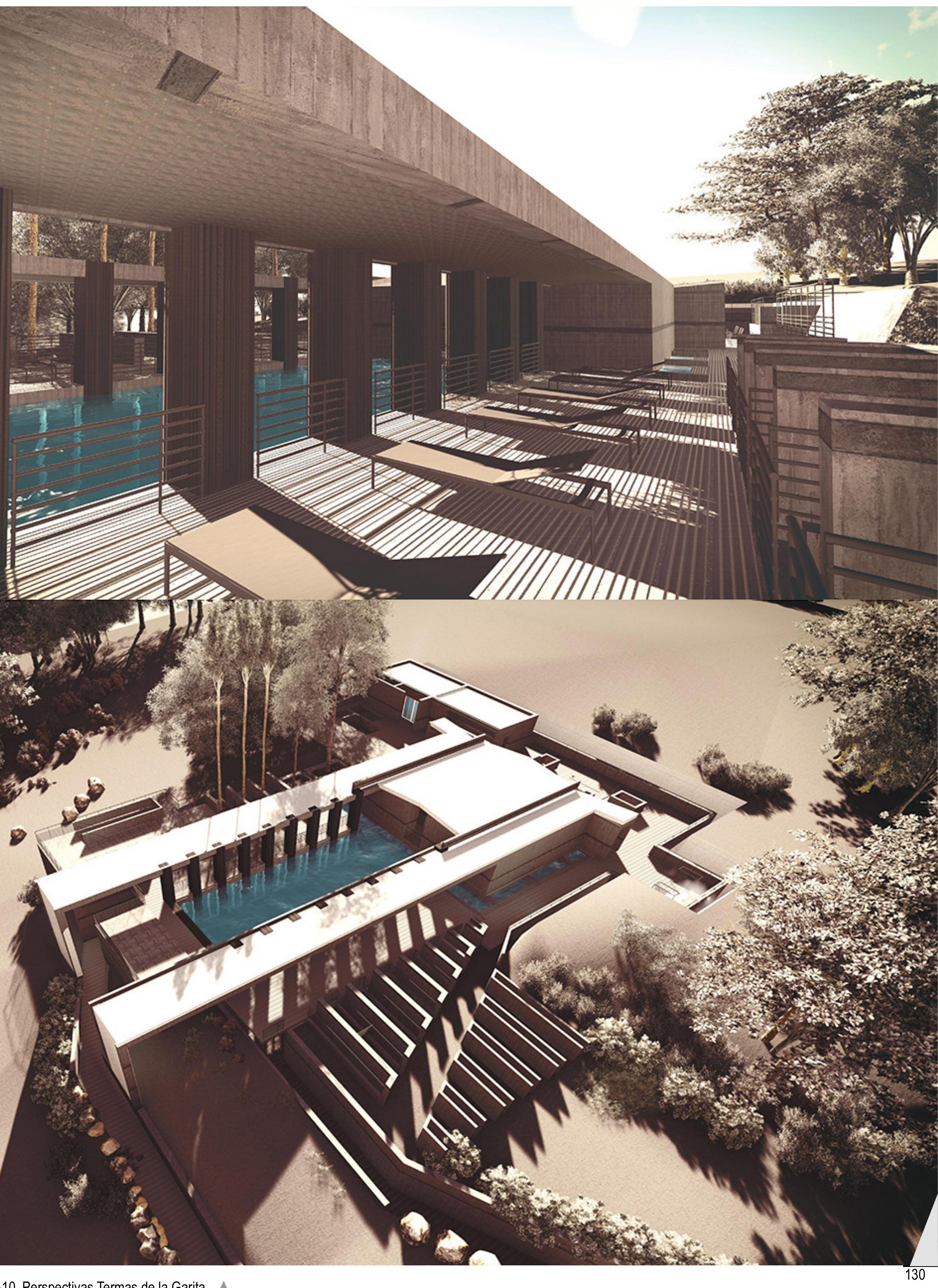

Figuras 9-10. Perspectivas. Termas de la Garita 


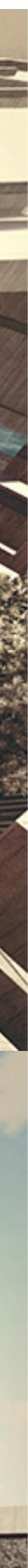

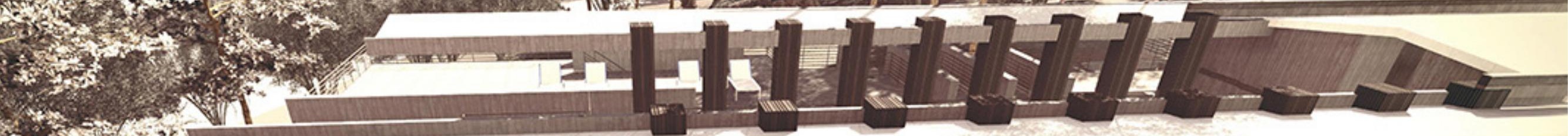
N 1

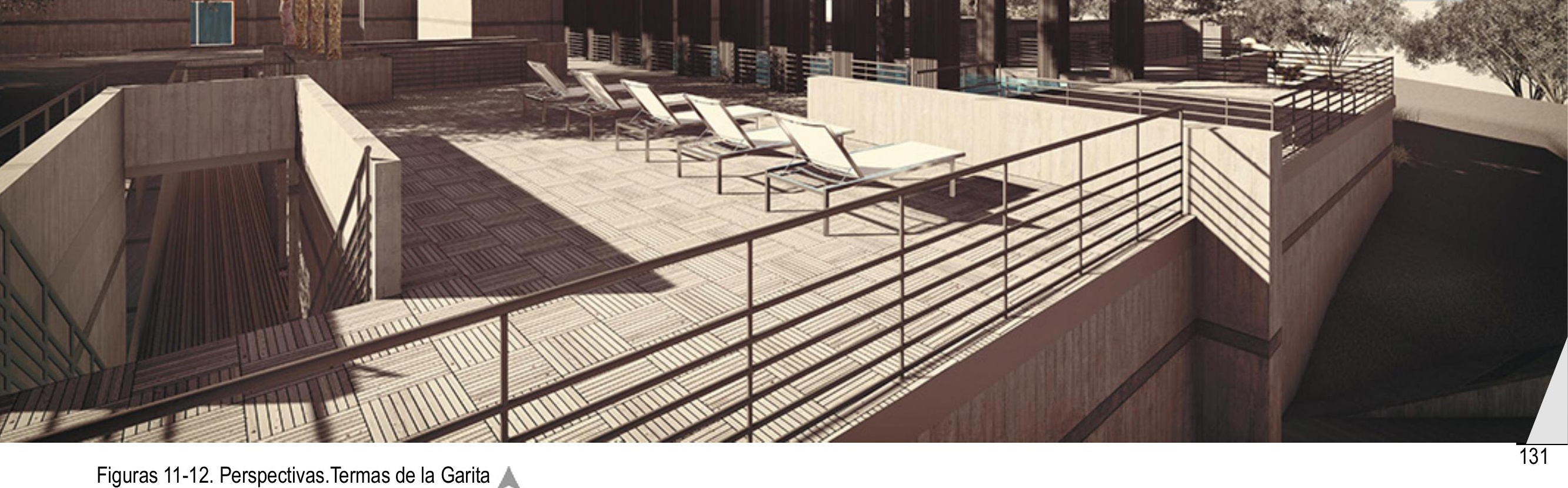

Figuras 11-12. Perspectivas. Termas de la Garita 


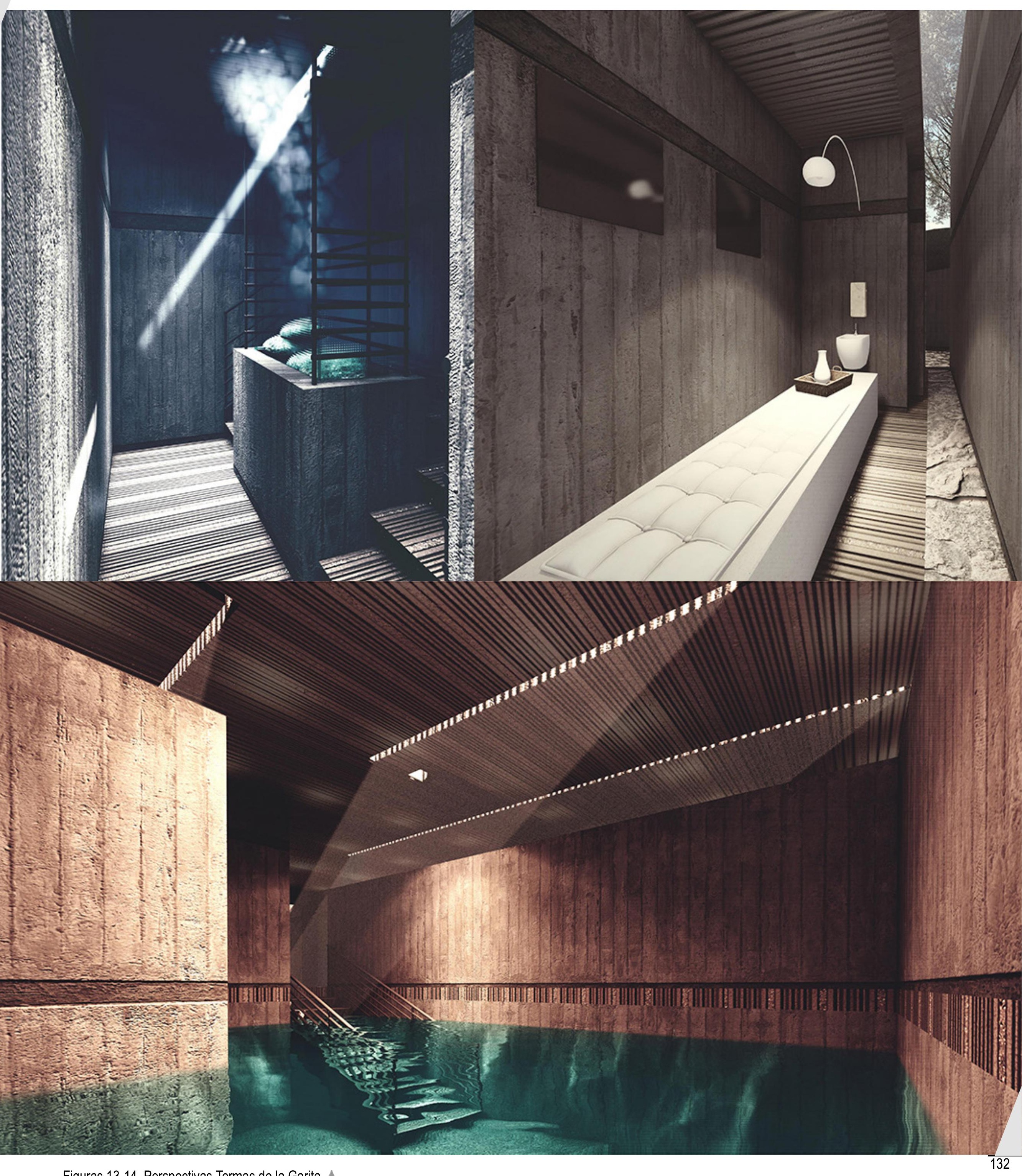

Figuras 13-14. Perspectivas. Termas de la Garita A 


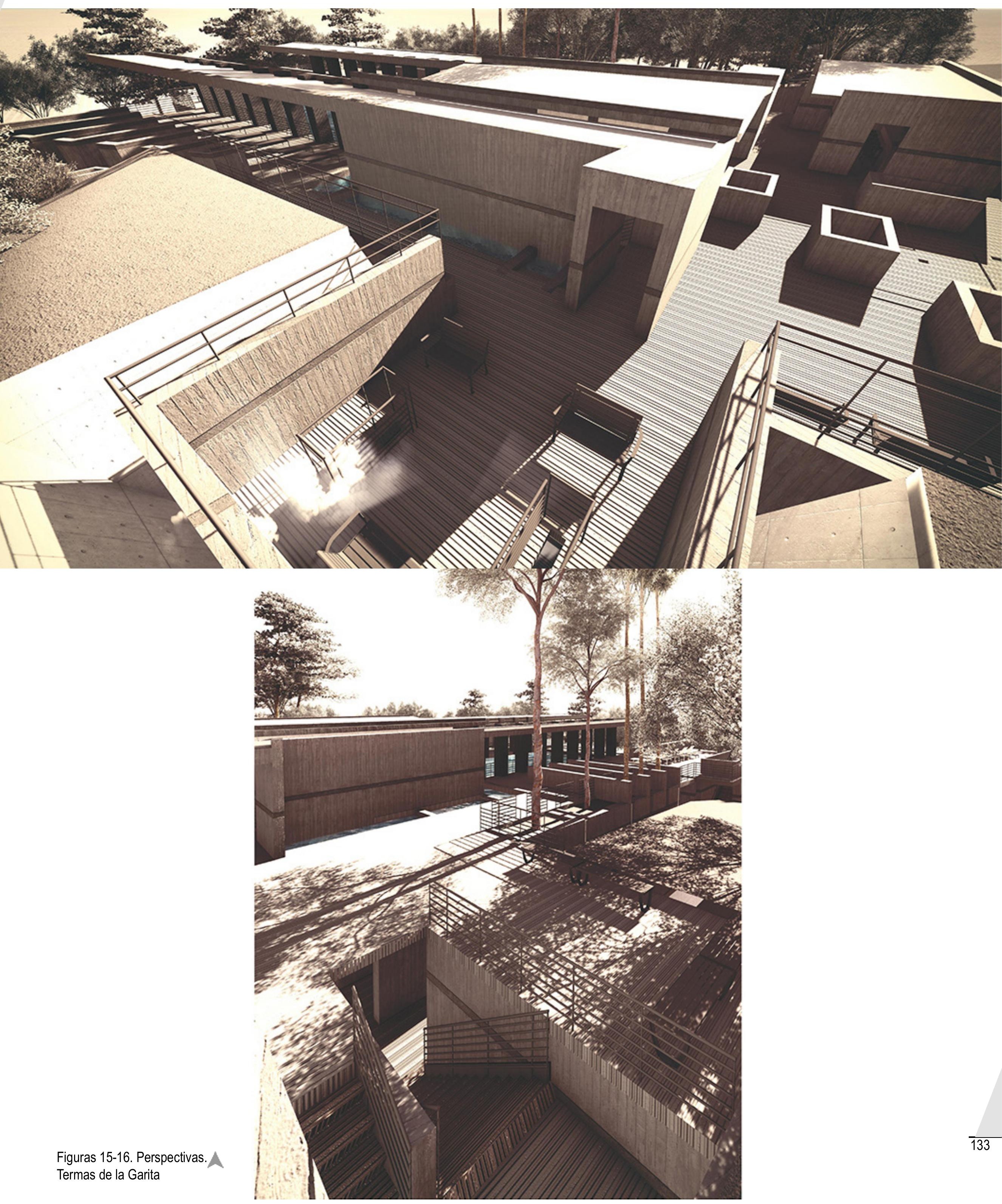

